\title{
Black and Ethnic Minority Academics' Perspectives on Intercultural Interaction in UK Universities: Beyond Racialization
}

\author{
Christian Atabong Nchindia ${ }^{1, *}$ \\ ${ }^{1}$ School of Education and Professional Development, University of Huddersfield, England, \\ United Kingdom \\ *Corresponding author: University of Huddersfield, 10 Queensgate, HD1 3DH Huddersfield, \\ United Kingdom. E-mail: Christian.AtabongNchindia@hud.ac.uk
}

Received: October 6, 2020 Accepted: November 5, 2020 Published: November 12, 2020

doi:10.5296/ije.v12i4.17790 URL: http://dx.doi.org/10.5296/ije.v12i4.17790

\begin{abstract}
Underpinned by frameworks of intercultural interaction, representation and discourse analysis, this research aimed to explore ambiguities and examine salient factors in intercultural interaction in UK universities, from the perspectives of four BAME lecturers and a lecturer from mainstream British culture. Informed by interpretive qualitative methodology and convenient sampling, data was collected through individual semi-structured interviews and the Nvivo software was used for analysis. Findings revealed that the experiences of academics in intercultural interaction with students were homogeneous across the board irrespective of their racial backgrounds. Despite a high level of intercultural awareness, some students and academics were unable to deconstruct stereotypes in cultural representation. It seems that universities do little to help academics and students develop and sustain intercultural awareness. There is hardly any systemic, structural, and coordinated approach in addressing cultural issues that may emerge in the classroom, as academics are left to figure it out by themselves. Some implications for policy in the higher education contexts were identified and recommendations made.
\end{abstract}

Keywords: intercultural interaction, intercultural communication, BAME academics, language, attainment gap, racialization 


\section{Introduction}

Albeit insufficient, a lot has been written on the experiences of Black, Asian, and Minority Ethnic (BAME) students in UK universities. Researchers seem to have neglected the experiences of BAME academics a great deal. Few studies conducted in this area were either focused on racialization (Sian, 2017), representativeness (Alexander \& Arday, 2015), or mobility (Bhopal, 2016). Research has established that there is a widening of attainment gap between white and BAME students in the UK at the disadvantage of the latter (Cotton el al, 2015; Richardson, 2015). Statistically, in 2017-18, the total white-BAME attainment gap was $13.2 \%$, which is the differences between the total white attainment, $80.9 \%$ and the total BAME attainment, 67.7\% (Universities UK, 2019). Among the many reasons identified for this gap was the lack of ethnic diversity among academic staff. "Having low numbers of BAME staff has been identified as limiting an institution's capability to address the attainment gap" (Universities UK 2019, p. 17). Sadif, a student from Kingston university supported this statement in an interview when he said, "Having a tutor from the same background as I gave me a sense of comfort. I was able to talk to them not only about academic issues, but also advice for me personally" (Universities UK, 2019, p. 20). It is in the context of the above assertion that this study is based. This paper is not concerned with the under representativeness of BAME academics but seeks to understand the experiences of the underrepresented in terms of their intercultural interaction with minority and mainstream students. To understand their experiences better in the context of intercultural interaction, the following research questions were formulated.

1. Do BAME academics have similar or different experiences teaching minority and mainstream students?

2. How do their own cultural background and identity affect their interaction with these groups of students?

3. What are their perspectives on intercultural group work and attainment gap between minority and mainstream students?

The interviewees were two black African male lecturers (one of them a professor), a black African female lecturer and an Asian male lecturer, all but two came from different universities in the North West of England. Alternative data was collected from one male white British lecturer who taught in the same university as the black female lecturer. This way, the researcher could compare views across 'mainstream' and 'BAME cultures'. The focus of this study was on how BAME academics perceived their intercultural interaction with students in terms of cultural diversity, language proficiency, and academic attainment. This study goes beyond hitherto research in this area which have focused largely on underrepresentation and racialization, although a few aspects of racialization were addressed in this paper. The first two factors (i.e. culture and language) have been established to play a direct role on minority student's academic achievement (Skutnabb-Kangas \& Cummins, 1988).

The main aim of the research was to explore ambiguities and examine salient factors in intercultural interaction in UK universities, from the perspectives of four BAME lecturers and 
a lecturer from mainstream British culture. The sub aim was to increase awareness of the problems that arise in intercultural communication between diverse groups of students and their lecturers, and how the latter can address the issues. In line with the aims, the objectives of this research were:

- To show the impact of communication on intercultural interaction among students, and between students and lecturers

- To identify implicit cultural assumptions held by both BAME and mainstream lecturers regarding intercultural interactions in UK universities

- $\quad$ To improve group cohesion among students by identifying and addressing the barriers that impede intercultural group interactions.

- Finally, to call attention to any counterproductive stereotypes and prejudices towards academics and students of other cultures.

\section{Literature Review}

The theoretical framework of this paper is underpinned by a combination of theories of intercultural interaction, representation, and discourse analysis. Intercultural interaction in this paper explores the influence of culture and culturally determined constructs on assumptions associated with interaction, teaching, and learning (Hinkel, 1999, p. 9). Culture underlies the ways in which language is used to create texts, whether these texts are written, audio-visual, or everyday interactions (Liddicoat \& Crozet, 2000, p. 2) between students and lecturers. Students and academics use language as a 'cultural tool' to mediate or connect with each other. However, a few factors can determine how participants in intercultural interaction understand each other's message, without resorting to miscommunication. One of these is the use of the linguistic code (Spencer-Oatley \& Franklin, 2009). If students or academics cannot speak English fluently, or if a proficient student or lecturer uses English in a copious way, it will be very difficult for them to understand each other (Spencer-Oatley \& Franklin, 2009). Another danger to intercultural interaction lies in interpreting the behavior of others in terms of the assumptions underlying one's own cultural views. This danger can be mitigated by what Antal and Friedman (2008) refer to as 'negotiating reality' (p. 363). Negotiating reality in intercultural interaction implies engaging with the other person in such a way as to seek to understand what might be driving his or her behavior and by helping that person to understand my behavior and expectations (Antal \& Friedman, 2008). To avoid breakdown in intercultural communication, students and academics may also be encouraged to acquire cultural literacy. This is the basic information one needs to know to carry on a reasonable conversation and to communicate or work with most people (Hui \& Chueng, 2014). Culturally literate students and academics may come to a position of empathy regarding each other's cultural identity (Halbert \& Chigeza, 2015, p. 157) in intercultural interaction. They would also be able to understand the complexity of cultures and that all cultures contain strengths, weaknesses and paradoxes, and the inevitable, ongoing, and complex nature of cultural change (Muller, 2006). Furthermore, they could analyze attributes of their own culture, and identify and deconstruct stereotypes; Lastly, they would be 
aware of cultural universals, internal (values-based) and external (lifestyle) components of culture, and the complex interrelationships of language and culture (Muller, 2006).

Representation in this study is focused on discourse analysis to examine how meanings are represented within narratives. This research thus explores how discourse shapes the structures of the context (Sian, 2017). Since speakers of different languages engage in an arbitrary division of reality (Thomas et al, 2004), it thus follows that every language could be a system of representation that mirrors and reinforces the worldview of its speakers (Thomas et al, 2004). Our perception of the world might also be socially constructed which highlights the notion of social representation, whereby culturally agreed upon explanations eventually come to be regarded as common-sense explanations (Holliday et al, 2010). We tend to use the words which are passed to us through our language and culture from an early age. A tendency Bourdieu referred to as the 'habitus.' According to Bourdieu (1968), individuals acquire a habitus very early in their cultural formation, inculcated largely through the pedagogical practices of the family. Once acquired, the habitus underlies and conditions all subsequent learning and social experience (Bourdieu, 1968, cited in Grenfell et al, 1998). Thus, there is no deliberate choice available to us about what a sign will stand for, and our decision to use the words given to us is not a conscious one (Kidd, 2015). Not only are what we say a result of habitus but also how we say them. A given language is never used in the same way by everyone of its speakers. Their accent and dialect may vary depending on their background, social status, geographical area, or region of origin (Jones, 2004). This variation in accent in Britain for example, might lead to stereotypes as people can be sometimes judged according to their accents. For instance, a speaker with the accent associated with Merseyside or West Midlands might be judged as being working class, while those with accents linked with the 'Home Counties' or Edinburgh might be placed at a higher position on the social scale (Jones, 2004, p. 135). In Wales, the accent of the English spoken in the city of Cardiff is different from the accent of Swansea, around 50kms along the coast (Austin \& Mathews, 2013). This variation of societal accents and dialects are reflected in the university classrooms and environments and can pose a real hurdle to intercultural communication. However, "we should be all encouraged to think about how our own contexts, ideas, prejudices, and partial worldviews, filter into and frame the representation we construct (Kidd, 2015, p. 22). Another aspect of discourse which has largely been ignored in studies of representation and language is silence. Kidd (2015) argues that it is easy to equate silence in representational terms with invisibility and lack of voice. However, such silences can be both a power marker and a sign of disempowerment. Therefore, in thinking about representation, educators should seek to investigate who is doing the silence and to what end (Kidd, 2015). On the other hand, it has been argued (Scallon, 1999) that modern classrooms and modes of communication have been superimposed on students and teachers, who hold assumptions handed down from the time of Socrates in the fifth, and Confucius in the sixth century BC. Although both Socrates and Confucius shared the educational ideal of upholding virtue, the former emphasized the virtue of thinking for oneself, while the latter preached the virtue of blind submission and rigid obedience to authority (Scallon, 1999). Understanding Socratic and Confucian discourse is vital in appreciating intercultural interaction and power relations between students and academics. Power relations, especially between the students and academics in this case, cannot be "established, consolidated nor implemented without the 
production, accumulation, circulation and functioning of a discourse" (Gordon, 1980, p. 93).

\section{Methodology}

The sampling method employed in this study was a non-probability sampling type called convenient sampling. A convenient sample is one that is available to the researcher by virtue of its accessibility (Bryman, 2004). Three of the five informants were known to the researcher, having met, or known them at conferences or cultural associations in Manchester. The other two were contacted through 'gatekeepers.' With this approach, the researcher made initial contacts with someone who knew them to establish contacts with them. One of the gatekeepers was an institution where the participant worked. This method of sampling was justifiable as the chances to collect data from these participants represented too good an opportunity to miss (Bryman, 2004). The data was collected over a period of four months as it was often difficult to successfully arrange and execute an interview because of respondents' tight schedules. At the start of each interview, respondents were briefed on the rationale for the research and were asked to sign an informed consent form, which they all did. All interviews were face-to-face, individual, and semi-structured, and each lasted for an hour. Open-ended questions were asked that allowed for a more interactive discussion between the interviewer and the respondents. This type of interview involves the implementation of several predetermined questions. These questions were typically asked of each interviewee in a systematic and consistent manner, but the interviewer occasionally digressed to probe far beyond the answers to his prepared questions (Berg, 2007, p. 95). This way, the interviewer was able to follow up ideas, clarify responses and investigate motives and feelings (Bell, 2005). Two audio recorders were used in each interview in case one failed during the process. Each recording was transcribed manually at the earliest opportunity and the data saved in a secured passworded device with the researcher having unique access to these data. In terms of ethical considerations, this research was conducted in a way that adhered to ethical requirements for doing research in the UK which is the legal jurisdiction of this project (Nchindia, 2020). These ethical obligations are embedded into the Data Protection Act (1998), the General Data Protection Regulations (2018), and the Ethical Guidelines for Educational research (McCulloch, 2018). Participants' right to confidentiality and anonymity was respected in this research as these are key considerations in research ethics. Anonymization was done by using imaginative names instead of respondents' real names even though their institutions were identified. It was necessary to mention the institutions for the purpose of cross-comparison. The table below shows participants' profile. 
Table 1. Participants' Profile

\begin{tabular}{|c|c|c|c|c|c|c|}
\hline Name & Institution & Gender & $\begin{array}{l}\text { Age } \\
\text { Range }\end{array}$ & $\begin{array}{l}\text { Teaching } \\
\text { Experience }\end{array}$ & Subject & Ethnicity \\
\hline Mr. Anton & $\begin{array}{l}\text { Bolton } \\
\text { University }\end{array}$ & Male & $35-45$ & 1 year & Nursing & $\begin{array}{l}\text { White } \\
\text { British }\end{array}$ \\
\hline Dr. Achal & $\begin{array}{l}\text { Liverpool } \\
\text { Hope } \\
\text { University }\end{array}$ & Male & $30-40$ & 5 years & $\begin{array}{l}\text { Business } \\
\text { Management }\end{array}$ & Asian \\
\hline $\begin{array}{l}\text { Dr. } \\
\text { Rosaline }\end{array}$ & $\begin{array}{l}\text { Bolton } \\
\text { University }\end{array}$ & Female & $40-50$ & 6 years & Law & $\begin{array}{l}\text { Black } \\
\text { African }\end{array}$ \\
\hline $\begin{array}{l}\text { Dr. } \\
\text { Stephen }\end{array}$ & $\begin{array}{l}\text { University of } \\
\text { Central } \\
\text { Lancashire }\end{array}$ & Male & $40-50$ & 5 years & $\begin{array}{l}\text { Construction } \\
\text { engineering }\end{array}$ & $\begin{array}{l}\text { Black } \\
\text { African }\end{array}$ \\
\hline $\begin{array}{l}\text { Prof. } \\
\text { Yuvin }\end{array}$ & $\begin{array}{l}\text { Manchester } \\
\text { University }\end{array}$ & Male & $50-60$ & 11 years & Law & $\begin{array}{l}\text { Black } \\
\text { African }\end{array}$ \\
\hline
\end{tabular}

\section{Data Analysis}

Detailed analysis was done using Nvivo software in which the 'explore' option was employed to run and produce coding queries, word frequency, word cloud, chart coding, hierarchy chart, and cluster analysis. Coding and analysis were done with each one of the interview datasets and all the above-mentioned queries were run for each dataset. This process took five days to complete, working eight hours per day analyzing each dataset. Tables, charts, and graphs were exported from Nvivo to excel and pictures exported and saved on the computer, and the ones used here were copied and pasted herein. The coded themes and subthemes in the tables that follow give us an insight into each respondent's file or 'story'.

Word frequency result for the first most reoccurring 1000 words in the interview with Mr. Anton, produced $32(2.28 \%)$ counts for the word student, followed by the word people with 28 $(2 \%)$ counts. This could indicate that the conversation was more focused on the students. A total of 21 themes were coded and several sub-themes, with each of the main themes producing a percentage coverage as shown in table 2 below. In the table, we can see that Mr. Anton covered a lot on 'how to be culturally sensitive' as a teacher, with $17.63 \%$ data coverage. 
Table 2. Coded Themes and Percentage Coverage for Mr. Anton

\begin{tabular}{|c|c|}
\hline Coding & $\begin{array}{l}\text { Percentage } \\
\text { coverage }\end{array}$ \\
\hline Codes $\backslash \backslash$ Accent and dialect as barrier $\backslash$ neck it down $\backslash$ consciously slow down my speech & $7.75 \%$ \\
\hline $\begin{array}{l}\text { Codes } \backslash \backslash \text { Accent and dialect can be an issue } \backslash \text { You must be careful } \backslash \text { cannot always be careful } \backslash I \\
\text { call everyone guys } \backslash \text { It is not sexist, but could be misinterpreted }\end{array}$ & $16.24 \%$ \\
\hline $\begin{array}{l}\text { Codes } \backslash \backslash \text { Accommodating cultures (2) } \backslash \text { do not bash in } \backslash \text { shout out before entering } \backslash \text { respect } \\
\text { privacy and dignity }\end{array}$ & $3.48 \%$ \\
\hline Codes $\backslash \backslash$ Accommodating cultures $\backslash$ females practice on females & $5.33 \%$ \\
\hline Codes $\backslash \backslash$ Background matters $\backslash$ pharmacists uncomfortable with touching & $3.27 \%$ \\
\hline Codes $\backslash \backslash$ Code-switching for a specific reason & $2.26 \%$ \\
\hline Codes $\backslash \backslash$ Cultural inclusivity in nursing $\backslash$ Explain to students & $2.17 \%$ \\
\hline Codes $\backslash \backslash$ Cultural interaction with colleagues $\backslash$ Understanding $\backslash$ develop banter & $3.74 \%$ \\
\hline Codes $\backslash \backslash$ Cultural issues in nursing $\backslash$ Touching people & $3.02 \%$ \\
\hline $\begin{array}{l}\text { Codes } \backslash \text { Culture as a habitus } \backslash \text { some cultures are straight-talking } \backslash \text { straight-talking can } \\
\text { upset } \mid \text { that's just the way they were brought up }\end{array}$ & $3.13 \%$ \\
\hline $\begin{array}{l}\text { Codes } \backslash \text { For example, some of the students must touch other people and because of cultural } \\
\text { issues, that can get a bit scary for them if they have not done it before. }\end{array}$ & $2.30 \%$ \\
\hline $\begin{array}{l}\text { Codes } \backslash \backslash \text { Home students speaking other languages } \backslash \text { Could be disruptive } \backslash \text { I won't understand } \\
\text { what they are saying } \backslash \text { Could help include L } 2 \text { students } \backslash \text { Could help student build bonds } \backslash \text { More } \\
\text { positives than negatives }\end{array}$ & $8.18 \%$ \\
\hline $\begin{array}{l}\text { Codes } \backslash \text { How can university develop intercultural communication } \backslash \text { provide diversity } \\
\text { training }\end{array}$ & $6.15 \%$ \\
\hline Codes $\backslash \backslash$ How to be culturally sensitive $\backslash$ Do not assume & $17.63 \%$ \\
\hline Codes $\backslash \backslash$ do not differentiate my lesson & $5.76 \%$ \\
\hline $\begin{array}{l}\text { Codes } \backslash \backslash \text { Language proficiency } \backslash \text { postgraduate are expected to be proficient } \backslash I \text { ask if they have } \\
\text { understood } \backslash \text { No one misunderstands because of languagelthey may not understand my } \\
\text { accent }\end{array}$ & $8.00 \%$ \\
\hline Codes $\backslash \backslash$ Multicultural group work in nursing practiceljust practicing on each other & $2.26 \%$ \\
\hline $\begin{array}{l}\text { Codes } \backslash \text { Teacher competence in foreign language can help } \backslash \text { The English expect everyone to } \\
\text { speak English } \backslash \text { linguistic imperialism \languages unhelpful for career path } \backslash \text { Laziness to learn } \\
\text { other languages } \backslash \text { languages should not be a prerequisite for teaching }\end{array}$ & $7.75 \%$ \\
\hline $\begin{array}{l}\text { Codes } \backslash \backslash \text { University's procedures gives teachers the responsibility to look at the } \\
\text { requirements of all students, whether that be cultural, linguistic, or disability for example. } \\
\text { We can never assume. We use the first couple of weeks to try and get to know the student }\end{array}$ & $1.95 \%$ \\
\hline $\begin{array}{l}\text { Codes } \backslash \backslash \text { Ways to encourage diversity } \backslash \text { BAME students, teachers and mainstream come } \\
\text { together } \backslash \text { Ask about each other's cultures } \backslash \text { Ask what upsets certain cultural groups } \backslash \text { English } \\
\text { not easily upset } \backslash \text { my actions might unintentionally upset others } \backslash \text { Sarcasm might upset some } \\
\text { people }\end{array}$ & $13.62 \%$ \\
\hline
\end{tabular}

Word frequency for 1000 most reoccurring words from Dr. Achal's data produced $44(3.45 \%)$ counts for the word students, followed by the word writing at $16(1.25 \%)$ counts. This could indicate that Dr. Achal was really concerned about students' writing. Again, the highest percentage coverage from his dataset as seen on the table below was $15.85 \%$ associated with 
the theme 'cultural issues in academic writing'. This confirms the earlier claim that he was deeply concerned with student's academic writing, especially some group of BAME students. All the data he provided was coded, giving him 100\% data coding.

Table 3. Coded Themes and Percentage Coverage for Dr. Achal

\begin{tabular}{|c|c|}
\hline Coding & $\begin{array}{l}\text { Percentage } \\
\text { coverage }\end{array}$ \\
\hline Cases $\backslash \backslash$ Interview 2 & $100.00 \%$ \\
\hline $\begin{array}{l}\text { Codes } \backslash \backslash \text { About translating text } \backslash \text { They speak to app and translate } \backslash \text { Writing style looks like } \\
\text { they're speaking } \backslash \text { Should fix it after translating } \backslash \text { Write and translate is better }\end{array}$ & $7.47 \%$ \\
\hline $\begin{array}{l}\text { Codes } \backslash \backslash \text { Academic dishonesty (2) } \backslash \text { Invite them for verbal feedback } \backslash \text { Lecturers are } \\
\text { helpless without provelNew Code }\end{array}$ & $8.13 \%$ \\
\hline Codes $\backslash \backslash$ Academic dishonesty $\backslash$ Some pay agents & $7.56 \%$ \\
\hline Codes $\backslash \backslash$ academics should speak up & $2.28 \%$ \\
\hline Codes $\backslash \backslash$ Attainment gap (2) \Indian students are better & $1.83 \%$ \\
\hline $\begin{array}{l}\text { Codes } \backslash \backslash \text { Attainment gap } \backslash \text { Local students write better } \backslash \text { Local students make grammatical } \\
\text { mistakes } \backslash \text { Local and African students are pared } \backslash \text { Chinese and S. Asians not much }\end{array}$ & $5.50 \%$ \\
\hline $\begin{array}{l}\text { Codes } \backslash \backslash \text { Cultural issues in academic writing } \backslash \text { citation and referencing } \backslash \text { developing } \\
\text { academic writing skills } \backslash \text { Plagiarism }\end{array}$ & $15.85 \%$ \\
\hline $\begin{array}{l}\text { Codes } \backslash \text { Cultural sensitivity } \backslash \text { Understand student's backgrounds } \backslash \text { South Asian students } \\
\text { have probs with ref } \backslash S \text {. Asian students do } 1 \text { block } 1 \text { citation } \backslash A \text { frican students generally } \\
\text { better } \backslash \text { West African students sound } \backslash \text { Colonial roots may be the reason } \backslash A \text { frican students } \\
\text { no problem with academic writing } \backslash \text { Chinese students a bit of a problem }\end{array}$ & $5.68 \%$ \\
\hline Codes $\backslash \backslash$ Embedding Intercultural competence in teaching $\backslash$ Feedback & $5.54 \%$ \\
\hline $\begin{array}{l}\text { Codes } \backslash \text { How to be culturally inclusive } \backslash \text { Students should give examples from home } \\
\text { countries } \backslash \text { Different modules different cultural issues }\end{array}$ & $5.03 \%$ \\
\hline $\begin{array}{l}\text { Codes } \backslash \backslash \text { How to develop intercultural competence } \backslash \text { You cannot teach it } \backslash \text { Guide sessions } \\
\text { with academics } \backslash \text { Through informal discussions }\end{array}$ & $1.64 \%$ \\
\hline $\begin{array}{l}\text { Codes } \backslash \backslash \text { Intercultural group work } \backslash \text { Students gravitate to own cultural group } \backslash \text { Pick and } \\
\text { mix them } \backslash \text { dissolve friendship groups } \backslash \text { Make them work with each other }\end{array}$ & $4.38 \%$ \\
\hline $\begin{array}{l}\text { Codes } \backslash \backslash \text { Other cultural aspects } \backslash \text { Chinese students do not ask questions } \backslash \text { Could be } \\
\text { shyness or language issue } \backslash \text { Some use translation in class Indian and African students } \\
\text { generally active } \backslash \text { Foreign students are respectful } \backslash \text { They address you with the title }\end{array}$ & $7.18 \%$ \\
\hline Codes $\backslash \backslash$ Poor academic practicelciting at the end of copied paragraph & $3.39 \%$ \\
\hline $\begin{array}{l}\text { Codes } \backslash \backslash \text { solution to poor academic practice } \mid \text { read students draft with them } \backslash \text { Correct } \\
\text { mistakes and give feedback } \backslash \text { Read second and third drafts }\end{array}$ & $2.02 \%$ \\
\hline Codes $\backslash \backslash$ Some students do not contribute $\mid$ remix them & $2.52 \%$ \\
\hline $\begin{array}{l}\text { Codes } \backslash \backslash \text { Support services (2) } \backslash \text { Writing mentors in library } \backslash \text { One-to-one sessions } \backslash \text { Taught } \\
\text { academic writing skills } \backslash \text { mentors' feedback to improve drafts } \backslash \text { mentors are natives }\end{array}$ & $5.17 \%$ \\
\hline Codes $\backslash \backslash$ Support services $\backslash$ cultural orientation $\backslash$ language exchange program $\backslash$ New Code & $2.27 \%$ \\
\hline Codes $\backslash \backslash$ Why students cheat & $2.18 \%$ \\
\hline
\end{tabular}


Table 4. Coded Themes and Percentage Coverage for Dr. Rosaline

\begin{tabular}{|c|c|}
\hline Coding & $\begin{array}{l}\text { Percentage } \\
\text { coverage }\end{array}$ \\
\hline $\begin{array}{l}\text { Codes } \backslash \backslash \text { About translation of text } \backslash \text { Might be due to language probs } \backslash \text { It changes the } \\
\text { structure of sentencellanguage impacts writing, thinking and structure }\end{array}$ & $2.50 \%$ \\
\hline $\begin{array}{l}\text { Codes } \backslash \backslash \text { About translation of texts } 2 \backslash \text { They are lost in translation } \backslash \text { Translation not } \\
\text { encouraged } \backslash \text { Think in English, write in English }\end{array}$ & $4.22 \%$ \\
\hline $\begin{array}{l}\text { Codes } \backslash \backslash \text { Academic dishonesty } \backslash \text { Students buying essays } \backslash \text { Lecturers are helpless } \backslash \text { Verbal test } \\
\text { can prove } \backslash \text { Students take offense when asked to take vivalChinese students are more } \\
\text { suspicious } \backslash \text { Politics in academia }\end{array}$ & $8.95 \%$ \\
\hline $\begin{array}{l}\text { Codes } \backslash \backslash \text { Accent and communication } \backslash \text { Some students complain about my accent } \backslash I \text { slow } \\
\text { down my speech }\end{array}$ & $4.30 \%$ \\
\hline Codes $\backslash \backslash$ Accent as barrier $\backslash$ Example of accent as barrier|Solution is use visual too & $4.83 \%$ \\
\hline $\begin{array}{l}\text { Codes } \backslash \text { Attainment gap and language (2) } \backslash \mathrm{L} 2 \text { students stick to topic } \backslash \text { English students } \\
\text { write too much }\end{array}$ & $2.44 \%$ \\
\hline Codes $\backslash \backslash$ Cheating $\backslash$ Cheaters blame it on language $\backslash$ Cheating is not cultural but individual & $6.77 \%$ \\
\hline Codes $\backslash$ Chinese students translate & $1.89 \%$ \\
\hline Codes $\backslash \backslash$ Cultural sensitivity $\backslash$ Know students background & $2.94 \%$ \\
\hline Codes $\backslash \backslash$ differences between Bolton and Manchester\Assignment versus exams & $6.59 \%$ \\
\hline Codes $\backslash$ disadvantage of not scrutinizing $\backslash$ bad educational outcome & $2.59 \%$ \\
\hline $\begin{array}{l}\text { Codes } \backslash \text { Dr. Rosaline's story- personal experiencelexample of lecturer-student } \\
\text { relations } \backslash \text { Easing cultural conflict }\end{array}$ & $3.14 \%$ \\
\hline $\begin{array}{l}\text { Codes } \backslash \backslash \text { Embedding intercultural competence } \backslash \text { Do extracurricular activities-football } \backslash D o \\
\text { an event in cultural clubs } \backslash \text { Do multifaith activities } \backslash \text { Lecturers and students' activities }\end{array}$ & $4.18 \%$ \\
\hline Codes $\backslash \backslash$ English is not my first language $\backslash I t$ is not a language assignment & $1.83 \%$ \\
\hline $\begin{array}{l}\text { Codes } \backslash \backslash \text { Example of accent as barrier } 2 \backslash \text { Murder or mother } \backslash \text { Borehole or well } \backslash \text { students } \\
\text { impatient with L2 students }\end{array}$ & $2.76 \%$ \\
\hline $\begin{array}{l}\text { Codes } \backslash \backslash \text { Examples of cultural differences } \backslash \text { Respect } \backslash \text { Hands up to speak } \backslash \text { Quiet versus noisy } \\
\text { class } \backslash \text { Chinese students \& Cameroon need prompting } \backslash \text { Come from authoritarian } \\
\text { society } \backslash \text { Brought up this way }\end{array}$ & $3.61 \%$ \\
\hline $\begin{array}{l}\text { Codes } \backslash \text { How to be culturally inclusive } \backslash \text { Give examples based on class } \\
\text { component } \mid \text { Examples from students' origin }\end{array}$ & $1.98 \%$ \\
\hline $\begin{array}{l}\text { Codes } \backslash \text { Intercultural group work ( } 2) \backslash \mathrm{I} \text { mix them up } \backslash \text { Problems working in groups } \backslash \text { People } \\
\text { don't turn up\not putting enough efforts } \backslash \text { some work more than others }\end{array}$ & $3.14 \%$ \\
\hline $\begin{array}{l}\text { Codes } \backslash \text { Intercultural group work } \backslash \text { students dependent on lecturers } \backslash \text { Students do not } \\
\text { interact with others } \mid \text { intercultural group work is importantlprepare them for the } \\
\text { workplacelprepares them for teamwork }\end{array}$ & $4.78 \%$ \\
\hline $\begin{array}{l}\text { Codes } \backslash \backslash \text { Racialization in university } \backslash \text { White lecturers mark down black students } \backslash \text { Black } \\
\text { lecturer stood up for back student } \backslash \text { Research has proven this discrimination } \backslash \text { White } \\
\text { lecturer not giving black student enough feedback } \backslash \text { University threaten to expel student } \\
\text { without procedure }\end{array}$ & $8.31 \%$ \\
\hline
\end{tabular}

Word frequency from Dr. Rosaline's dataset for the first 1000 reoccurring words produced the word students at the top with $63(4.29 \%)$ counts, followed by the word language with 28 $(1.90 \%)$ counts. We can see a pattern developing among all respondents and the data seem to indicate that Dr. Rosaline said a lot about students and language. However, her highest coding percentage coverage as shown in the next table, was associated with the theme 'academic 
dishonesty' with $8.95 \%$.

Table 5. Coded Themes and Percentage Coverage for Dr. Stephen

\begin{tabular}{|c|c|}
\hline Coding & $\begin{array}{l}\text { Percentage } \\
\text { coverage }\end{array}$ \\
\hline $\begin{array}{l}\text { Codes } \backslash \backslash \text { About translating texts } \backslash \text { Not good for students } \backslash \text { They need to learn English } \backslash \text { English } \\
\text { is why some are here } \backslash \text { I examine my own English } \backslash \text { I act as role model in way of } \\
\text { speaking } \backslash \text { Don't like texts translation } \backslash \text { Work can be proofread } \backslash \text { They should have language } \\
\text { skills } \backslash \text { English good for job prospect } \backslash \text { Certificate is in English }\end{array}$ & $4.83 \%$ \\
\hline $\begin{array}{l}\text { Codes } \backslash \backslash \text { Accent as barrier } \backslash \text { Native students' complaint } \backslash \text { It's not a problem } \backslash \text { It's a problem } \\
\text { with teacher complaint about students' accents } \backslash \text { Tutors supposed to support } \\
\text { students } \backslash \text { Problem when they can't understand you }\end{array}$ & $5.69 \%$ \\
\hline $\begin{array}{l}\text { Codes } \backslash \text { Cheating } \backslash \text { Some rich foreign students pay agents } \backslash \text { They score higher in assignment } \\
\text { than exams }\end{array}$ & $4.67 \%$ \\
\hline $\begin{array}{l}\text { Codes } \backslash \text { Cultural conflicts } \backslash I \text { 've been cautious } \backslash \text { Know students backgrounds } \backslash \text { Know your } \\
\text { cohorts } \backslash \text { Don't invade personal space } \backslash \text { Keep your distance }\end{array}$ & $2.87 \%$ \\
\hline $\begin{array}{l}\text { Codes } \backslash \text { Cultural sensitivity } \backslash \text { Respect their religion } \backslash \text { Use examples with care } \backslash \text { Do not offend } \\
\text { students } \backslash \text { Do not compromise quality }\end{array}$ & $4.66 \%$ \\
\hline $\begin{array}{l}\text { Codes } \backslash \backslash \text { Culturally inclusive teaching (2) } \backslash \text { You must be inclusive } \backslash \text { Address every student's } \\
\text { needs } \backslash \text { Provide required support } \backslash \text { whether disabled or dyslexic } \backslash \text { adjust your slides } \backslash \text { change } \\
\text { slide colors if necessary }\end{array}$ & $4.85 \%$ \\
\hline $\begin{array}{l}\text { Codes } \backslash \backslash \text { Culturally inclusive teaching } \backslash \text { Ask questions } \backslash \text { Bring level down } \backslash \text { Extend hand of } \\
\text { support } \backslash \text { Meet weaker students for a chat } \backslash \text { Do not sacrifice quality }\end{array}$ & $6.07 \%$ \\
\hline $\begin{array}{l}\text { Codes } \backslash \backslash \text { Developing intercultural competence } \backslash \text { Universities are big places } \backslash \text { Difficult to } \\
\text { organize } \backslash \text { Provide financial support } \backslash \text { Work with team leaders } \backslash \text { Organize social events } \backslash N e v e r \\
\text { socialized with colleagues }\end{array}$ & $6.34 \%$ \\
\hline Codes $\backslash \backslash$ English students have language issues $\backslash$ Example English students language issues & $2.87 \%$ \\
\hline Codes $\backslash \backslash$ Example of accent as barrier & $7.16 \%$ \\
\hline Codes $\backslash \backslash$ Example of failure in inclusivity & $3.38 \%$ \\
\hline $\begin{array}{l}\text { Codes } \backslash \text { Example of intercultural activity } \backslash \text { Trip to West Lake CumbrialStudents bond } \\
\text { together }\end{array}$ & $6.97 \%$ \\
\hline Codes $\backslash \backslash$ Lazy student's complaint most & $2.52 \%$ \\
\hline $\begin{array}{l}\text { Codes } \backslash \backslash \text { Multicultural group work (2) } \backslash \text { Same culture group together } \backslash \text { Move them } \\
\text { around } \backslash \text { Do intergroup report back }\end{array}$ & $3.85 \%$ \\
\hline $\begin{array}{l}\text { Codes } \backslash \text { Racialization } \backslash \text { complaining students may not be racistlcomplaining students are } \\
\text { lazy } \backslash \text { Mostly 1st year students complain } \backslash \text { student backgrounds may influence } \\
\text { complaints } \backslash \text { Students used to a particular accent } \backslash \text { New lecturer are mostly the } \\
\text { victims } \backslash \text { Students already know old lecturers } \backslash \text { Students are used to old lecturers }\end{array}$ & $4.78 \%$ \\
\hline $\begin{array}{l}\text { Codes } \backslash \backslash \text { Reasons why students cheat } \backslash \text { No prior knowledge of program studied } \backslash \text { Poor } \\
\text { English } \backslash \text { Universities recruit students for the money }\end{array}$ & $3.57 \%$ \\
\hline $\begin{array}{l}\text { Codes } \backslash \text { Solutions to cheating problem } \backslash \text { Solutions to cheating problem } 2 \backslash \text { Introduce a } \\
\text { viva } \backslash 15 \text { minutes presentation } \backslash \text { Standing panel to verify work }\end{array}$ & $2.67 \%$ \\
\hline $\begin{array}{l}\text { Codes } \backslash \backslash \text { Structural constraints } \backslash \text { No rigid structure } \backslash \text { You don't just care } \backslash \text { You just do your } \\
\text { job } \backslash \text { No suggestion boxes } \backslash \text { No places to complain } \backslash \text { Support not close enough }\end{array}$ & $3.21 \%$ \\
\hline $\begin{array}{l}\text { Codes } \backslash \text { Support services (2) } \backslash 3 \text { levels of support } \backslash F \text { oundation route } \backslash \text { WISER- one-to-one } \\
\text { talk } \backslash \text { One-to-one with tutor }\end{array}$ & $6.23 \%$ \\
\hline $\begin{array}{l}\text { Codes } \backslash \text { The role of accent } \backslash I \text { don't understand some students } \backslash \text { Poor accent prevents students } \\
\text { from talking } \backslash \text { Frustrating for the teacher }\end{array}$ & $7.87 \%$ \\
\hline
\end{tabular}


Dr. Stephen's dataset produced the word students as the most frequently reoccurring word at $81(4.59 \%)$ counts, followed by the word English at $25(1.42 \%)$ counts. Once again, we see that the pattern continues with a high focus on student and English during this interview. The table below shows that the theme with the highest percentage coverage in this dataset was 'the role of accent' with $7.87 \%$ coverage. The respondent emphasized on the importance of speaking clearly when interacting with students.

Lastly, word frequency report for Professor Yuvin's dataset produced the word students the most at $70(5.19 \%)$ counts, followed by the word English at $20(1.48 \%)$ counts. This pattern is consolidated across all five respondents. Regarding percentage coverages, the theme 'different academic cultures' stood out in Prof. Yuvin's dataset, scoring a percentage coverage of $12.55 \%$ as shown in the table below.

Table 6. Coded Themes and Percentage Coverage for Prof. Yuvin

\begin{tabular}{|c|c|}
\hline Coding & $\begin{array}{l}\text { Percentage } \\
\text { coverage }\end{array}$ \\
\hline $\begin{array}{l}\text { Codes } \backslash \backslash \text { About translating texts } \backslash \text { writing in English } \backslash \text { reflecting in mother tonguelit } \\
\text { doesn't make sense sometimes }\end{array}$ & $5.36 \%$ \\
\hline Codes $\backslash$ lacademic cheatinglinduct students on plagiarism & $3.35 \%$ \\
\hline Codes $\backslash \backslash$ Accent as barrierladjust accent to make students understand & $5.05 \%$ \\
\hline Codes $\backslash \backslash$ Actions against cheating $\backslash$ disciplinary procedures $\backslash$ repeat the assignment & $3.07 \%$ \\
\hline $\begin{array}{l}\text { Codes } \backslash \backslash \text { Adjust students by-|preparing detailed handouts } \backslash \text { Point to different } \\
\text { chapters } \backslash \text { Point to must-read chapters } \backslash \text { Not many referrals } \backslash \text { Easy to understand books }\end{array}$ & $10.13 \%$ \\
\hline $\begin{array}{l}\text { Codes } \backslash \backslash \text { Attainment gap } \backslash \text { gap in language proficiency } \backslash N o \text { gap in academic } \\
\text { achievement } \backslash \text { no gap in intellectual capacity }\end{array}$ & $3.39 \%$ \\
\hline Codes $\backslash \backslash$ Chinese students reproduce & $5.03 \%$ \\
\hline $\begin{array}{l}\text { Codes } \backslash \backslash \text { Culturally inclusive teaching (2) \organize seminars } \text { group students } 10-12 \\
\text { max } \backslash \text { talk \& share with each other } \backslash \text { share diff. experiences } \backslash \text { seminar is very inclusive }\end{array}$ & $7.31 \%$ \\
\hline $\begin{array}{l}\text { Codes } \backslash \backslash \text { Culturally inclusive teaching ( } 3) \backslash \text { Talk so students understand } \backslash \text { Sing from same } \\
\text { song sheet } \mid \text { pace the lecturelask questions }\end{array}$ & $3.84 \%$ \\
\hline Codes $\backslash \backslash$ Culturally inclusive teaching $\backslash$ consider L2 students when teaching & $7.21 \%$ \\
\hline $\begin{array}{l}\text { Codes } \backslash \backslash \text { Developing intercultural competencelwe organize eventsle.g. postgraduate } \\
\mathrm{BBQ} \backslash \text { postgraduate lunch } \backslash \text { postgraduate dinnerldifferent law societieslvibrant } \\
\text { international bodyleasy to interact }\end{array}$ & $9.41 \%$ \\
\hline $\begin{array}{l}\text { Codes } \backslash \text { Different academic culturelstyle of teaching } \backslash \text { students' idea of } \\
\text { essaylindependent thinking } \backslash \text { not reproduction }\end{array}$ & $7.13 \%$ \\
\hline Codes $\backslash \backslash$ Different academic cultures & $12.55 \%$ \\
\hline Codes $\backslash \backslash$ Encouraging intercultural interactions & $6.69 \%$ \\
\hline Codes $\backslash$ How to earn marks $\backslash$ read beyond class work & $2.41 \%$ \\
\hline $\begin{array}{l}\text { Codes } \backslash \text { Intercultural group work } \backslash 150 \text { nationalities in law school } \backslash \text { consider diff. } \\
\text { backgrounds } \backslash \text { some students do not talk } \backslash \text { no trust in self } \mid \text { no believe in self } \mid \text { fear of being }\end{array}$ & $4.42 \%$ \\
\hline
\end{tabular}


wrong

Codes $\backslash \backslash$ No complaint from students $\backslash$ might be being polite $\backslash$ might be understanding

me

Codes $\backslash$ Observation of colleagues

$4.20 \%$

Codes $\backslash \backslash$ Reasons students cheat $\backslash n$ ot understanding context $\backslash$ students from abroad may

cheat $\backslash$ poor citation $\backslash$ copying portions of work $\backslash$ Proposal stealing by $\mathrm{PhD}$ students $\backslash \mathrm{We}$ don't admit them $\backslash$ No essaying buying here

Codes $\backslash$ Support services $\backslash$ Official and unofficial $\backslash$ in-sessional courselacademic advising

A cluster analysis for both word and coding similarities across all five cases was produced using Pearson correlation coefficient similarity metrics. This cluster analysis indicated that there was a significant correlation between coded items across the cases which cannot be displayed here because of lack of space. We can see from figure 1 overleaf that similar words and themes that converged are color-coded with the same colors. The pattern tells us that there was much convergence in the ideas expressed by all respondents though they were all interviewed separately. Table 7 and figure 2 overleaf further support these correlations. Although the correlations in table 7 are not strong because they are all below coefficient 1 , there still exist reasonable correlations across respondents' files as emphasized on figure 2 overleaf.

Table 7. Correlation between Respondents' Files

\begin{tabular}{|c|c|c|c|c|c|}
\hline \multicolumn{3}{|c|}{ File A } & \multicolumn{2}{|l|}{ File B } & \multirow{2}{*}{$\begin{array}{c}\text { Pearson correlation } \\
\text { coefficient }\end{array}$} \\
\hline $\begin{array}{l}\text { Files } \backslash \backslash \text { Interview2 } \\
\text { Liverpool Hope uni }\end{array}$ & $\begin{array}{c}\text { Dr. } \\
\text { versity }\end{array}$ & Achal, & $\begin{array}{l}\text { Files } \backslash \text { Interview1, } \\
\text { Bolton university }\end{array}$ & Anton, & \\
\hline $\begin{array}{l}\text { Files } \backslash \backslash \text { Interview1, } \\
\text { Bolton university }\end{array}$ & Mr. & Anton, & $\begin{array}{l}\text { Files } \backslash \backslash \text { Interview 5, Prof } \\
\text { Manchester university }\end{array}$ & Yuvin, & -0.232115 \\
\hline $\begin{array}{l}\text { Files } \backslash \backslash \text { Interview2 } \\
\text { Liverpool Hope uni }\end{array}$ & $\begin{array}{c}\text { Dr. } \\
\text { versity }\end{array}$ & Achal, & $\begin{array}{l}\text { Files } \backslash \backslash \text { Interview 5, Prof } \\
\text { Manchester university }\end{array}$ & Yuvin, & -0.232115 \\
\hline $\begin{array}{l}\text { Files } \backslash \backslash \text { Interview4 } \\
\text { UCLAN, Preston }\end{array}$ & Dr. & Stephen, & $\begin{array}{l}\text { Files } \backslash \backslash \text { Interview1, } \\
\text { Bolton university }\end{array}$ & Anton, & -0.23812 \\
\hline $\begin{array}{l}\text { Files } \backslash \backslash \text { Interview4 } \\
\text { UCLAN, Preston }\end{array}$ & Dr. & Stephen, & $\begin{array}{l}\text { Files } \backslash \backslash \text { Interview2 Dr. } \\
\text { Liverpool Hope university }\end{array}$ & Achal, & -0.23812 \\
\hline $\begin{array}{l}\text { Files } \backslash \backslash \text { Interview4 } \\
\text { UCLAN, Preston }\end{array}$ & Dr. & Stephen, & $\begin{array}{l}\text { Files } \backslash \backslash \text { Interview } 5, \text { Prof } \\
\text { Manchester university }\end{array}$ & Yuvin, & -0.251233 \\
\hline $\begin{array}{l}\text { Files } \backslash \backslash \text { Interview3, } \\
\text { Bolton university }\end{array}$ & Dr. & Rosaline, & $\begin{array}{l}\text { Files \\
Interview1, } \quad \mathrm{Mr} . \\
\text { Bolton university }\end{array}$ & Anton, & -0.26192 \\
\hline $\begin{array}{l}\text { Files } \backslash \backslash \text { Interview3, } \\
\text { Bolton university }\end{array}$ & Dr. & Rosaline, & $\begin{array}{l}\text { Files } \backslash \text { Interview2 Dr. } \\
\text { Liverpool Hope university }\end{array}$ & Achal, & -0.26192 \\
\hline $\begin{array}{l}\text { Files } \backslash \backslash \text { Interview3, } \\
\text { Bolton university }\end{array}$ & Dr. & Rosaline, & $\begin{array}{l}\text { Files } \backslash \backslash \text { Interview 5, Prof } \\
\text { Manchester university }\end{array}$ & Yuvin, & -0.276344 \\
\hline $\begin{array}{l}\text { Files } \backslash \backslash \text { Interview4 } \\
\text { UCLAN, Preston }\end{array}$ & Dr. & Stephen, & $\begin{array}{l}\text { Files } \backslash \backslash \text { Interview3, Dr. } \\
\text { Bolton university }\end{array}$ & Rosaline, & -0.283493 \\
\hline
\end{tabular}




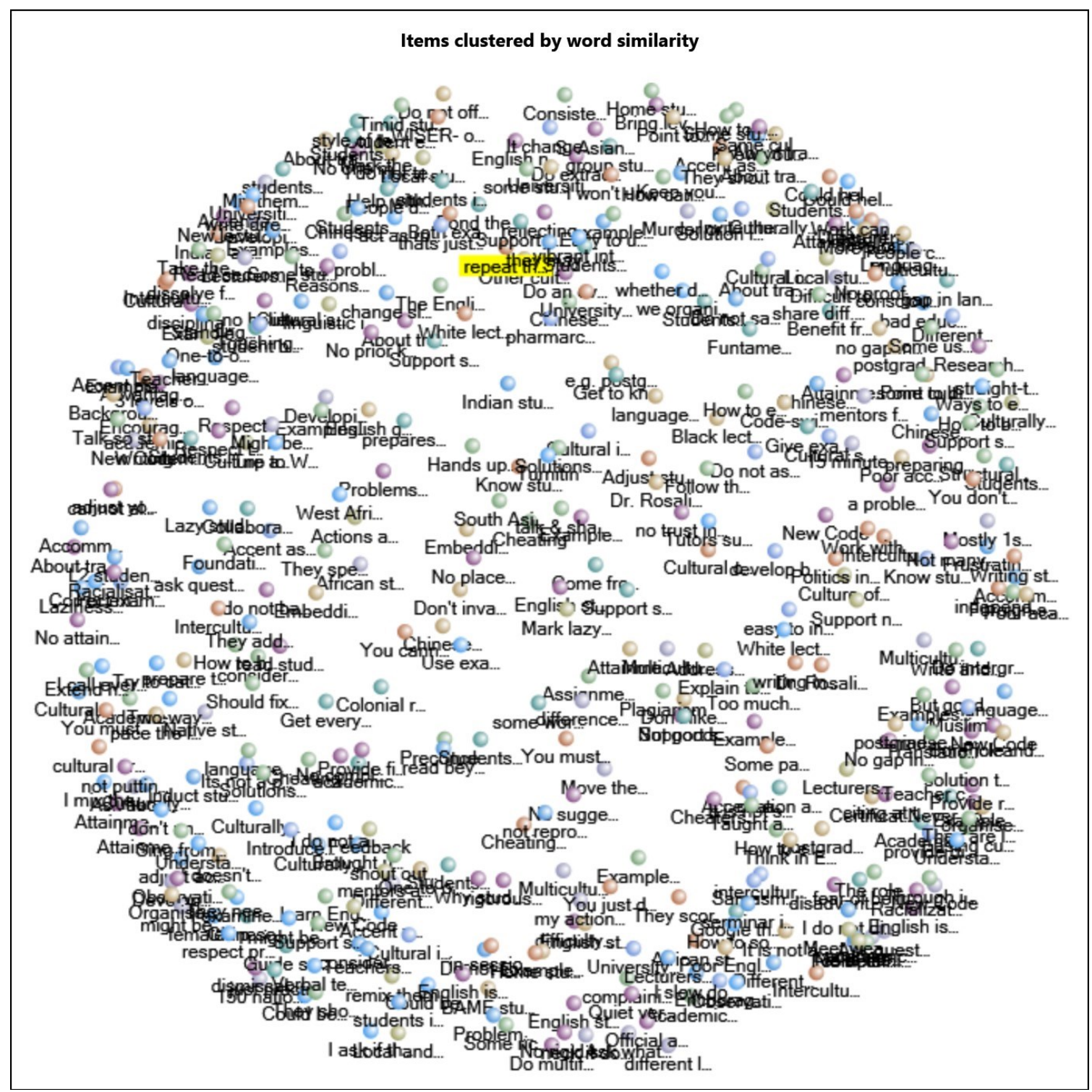

Figure 1. Items Coded by Word and Themes Similarities across all Five Respondents

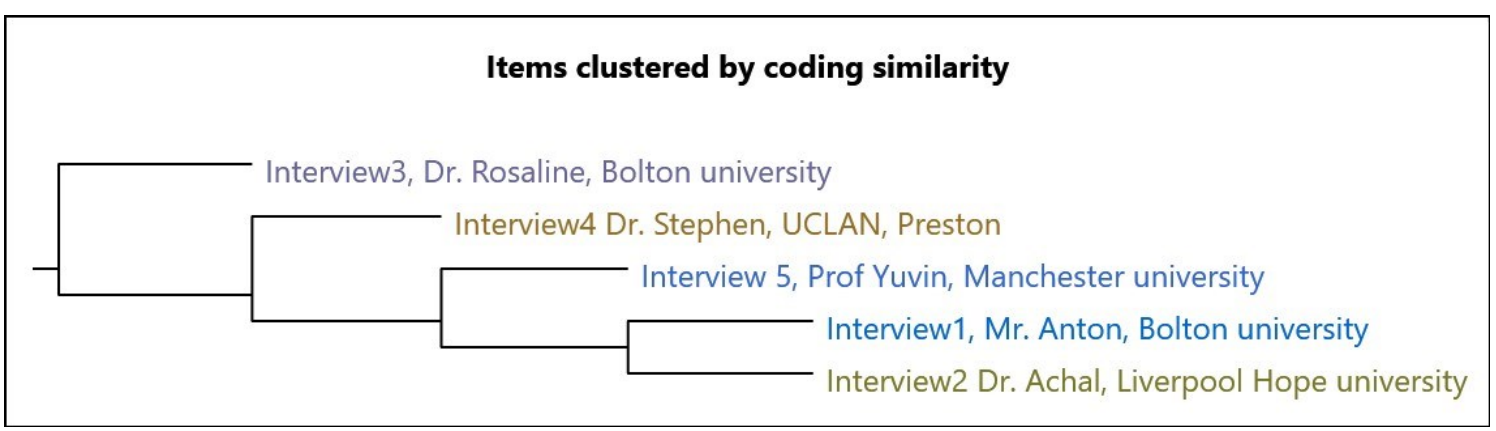

Figure 2. Items Clustered by Coding Similarities across all Respondents' File 
In figure 2, similarity in colors indicates convergence in opinions between respondents. Dr. Stephen and Dr. Achal have similar colors, same as Dr. Yuvin and Mr. Anton. However, Dr. Rosaline's color is slightly distinct. Giving that she was the only woman among respondents, we can argue that there was something about her opinion that diverged away from those of the men, invoking a gender-based difference here. The huge data that she provided on racism, which none of the men talked about might have caused this divergency to occur.

\section{Results}

Considering the themes and subthemes generated from data analysis, the results of this study have been grouped into three main categories which I prefer to call contexts. These are context of culture, context of language and context of academic attainment.

\subsection{Context of Culture}

All five academics in this study demonstrated a high degree of intercultural awareness which is the third and most advanced level of Baker's (2011) Intercultural awareness model. They seem to be all very aware of the role of culture in intercultural communication when interacting with students. This is what Mr. Anton who teaches a practical nursing course said which indicates intercultural awareness.

Our students are expected to practice on each other. When you first meet the students in the classroom on day one, you don't know how that is going to go. For example, some of the students have to touch other people and because of cultural issues, that can get a bit scary for them if they have not done it before (Mr. Anton).

Apart from being culturally sensitive, this respondent was capable of mediating and negotiating between different emergent culturally and contextually based communication modes (Baker 2011) when dealing culturally diverse students in his course.

...So, they will be doing it on practically bare chest. We have to basically explain to them that whatever your culture is, whatever your background is, if you want to do this in real life, this is how you do it. And because people come up to the course for a specific reason, they kind of know that before they get on it (Mr. Anton)

The four BAME academics also expressed cultural sensitivity and awareness in intercultural interaction, with each one of them presenting their views in different ways as stated below:

You really must understand what background they are coming from. And through my experience, I come to know what kind of weaknesses every culture will have- Dr Achal.

It is very important to consider where they are coming from especially with me being a foreigner as well...For example, respect. We come from a culture where we have huge respect for elders, where age and position are very important. So, the way you address people or speak to them is different (Dr Rosaline). 
I have not encountered conflicts with students based on cultural differences because I have been very cautious. The first thing I do when I meet my students for the first time is that I want to look at their backgrounds. And, once I am sure about their background, I want to know how to navigate myself around it (Dr Stephen).

We are also mindful that, sometimes some students find it challenging to engage in conversations with strangers - that is, people they meet here (Prof. Yuvin).

However, the mismatches in students' previous academic culture with that of the UK seemed to be more evident than issues with religious affiliation or cultural habitus (Bourdieu 1968, cited in Grenfell et al, 1998). These mismatches depended on the type of course taught or being studied. We have noted how nursing students ought to practice on each other and that can be problematic to some religions. BAME academics explained how they must be careful with the kind of examples that they give in class during lectures. In his course for instance, a BAME lecturer in this study wanted to show students an example of hazardous chemicals in a TANI industry in India. The picture was very unpleasant. There was an Indian student in class. Therefore, he had to look for the best way to present that picture without offending the student and without affecting the quality of his teaching. Another BAME lecturer explained how one of her students was offended because of the example she gave which according to her was a genuine observation. She had said she does not like cats, but one student took it so personal that she went to her office and asked her why she said she does not like cats? "What has cats done to you?" The student questioned her. She was surprised that it was so important to the student for her to like cats. "I said I don't like cats" the lecturer insisted. She went further to explain to the student that she was sure there are people who do not like dogs, or elephants but she does not like cats. The student left obviously disappointed and took it so personal. Since then, the lecturer has been very careful with what she says or the examples she gives in class"even something as basic as that", she exclaimed! Again, another student took offence with one BAME lecturer in this study when the latter told her that her writing style was like she was speaking, and that she writes just the way she speaks. He had suspected the student of having spoken to a device and translated that into written text, but the student got offended.

Academic cultural mismatches were particularly evident in students' writing. BAME academics in this study reported that some students find it difficult to write an essay and that could be attributed to the different perspectives on how to write essays that students bring from their home countries. According to an Asian academic, students from South Asia have problems with referencing and citation. They do what he calls "one block one citation", but students from Africa are generally better with strong writing skills which just need refining. He also reported that Chinese students can have a bit of a problem in terms of expression and academic writing. The law professor supported this claim but insisted that the problem was not limited to Chinese students. He reported that students from other parts of the world tend to feel that they can simply reproduce what were taught in class as if "we are testing their memory". This attitude betrays the whole purpose of university education, which is to help students develop independent thinking, he posits.

The culture of cheating or academic dishonesty emerged strongly in the data (Marsh, 2019). 
The practice of students buying essays from agencies that are specialized in providing these services was described by one BAME academic as "open and shameless". The respondent could tell when students have bought essays or written the essays themselves. For example, a student who sends him an email with so many grammatical errors will subsequently submit a flawless essay. This type of student will not submit drafts for correction prior to final submission neither will they use the core books of the course nor the books in the library; and the authors cited are uncommon. Other academics echoed similar experiences with students cheating as quoted below:

These days foreign students come in with a lot of money. And for some of these students, unfortunately for universities, they have got the money to even pay people to do the work for them. If you look at how the student behaves in class, how they respond to certain questions that you've given them, you can straightaway know that what they are giving can never be from them (Dr Stephen).

A student who is going to cheat will cheat, so those who cheat do so and blame it on the language. They do not try. They cheat and blame it on the language... Yes, we had one student who was not a very bright student. In the second year, his dissertation was just too good and couldn't have been written by him, and there was nothing we could do (Dr Rosaline)

All BAME academics in this study who have had experiences with students cheating in this way, also expressed their frustration and impotence in dealing with the issue. Universities require the lecturer to prove beyond doubts that the student has cheated and if you cannot prove it, the appeal panel will rule in favor of the student. As a solution, all academics interviewed agreed that giving students a viva exam to verbally defend their essays in front of a panel could help, but even this cannot guarantee that students will not cheat. The reasons why some students cheat was identified. According to Dr Achal, students come in from all sort of backgrounds who have no clue as to how to start writing, which topic to pick, or how to interview people, so they pay five or six hundred to anyone to write it up for them. Dr Stephen on his part explains that some students get into the program without the requisite knowledge. Furthermore, some have got the knowledge, but their English is poor. The issue of poor English being responsible for cheating habits should not be an excuse as most universities offer proofreading services which can help correct grammatical errors as my data suggest. Data also suggest that some universities take this problem seriously than others. Manchester university for instance have a "very rigorous way to check essays", according to Professor Yuvin. Students are warned about the impact of plagiarism at induction and are given handouts to explain this, but one in fifty still tries to cheat.

When we identify them, there are disciplinary procedures that the student goes through. For example, a student who has copied, let's say you have submitted your essay, and we see that 30 percent is not your work, then we will invite you for a disciplinary hearing. When we interview and hear your viewpoints about that, the different procedures will be put in place. For example, they will give you a zero, then you will have to do it again and resubmit. But if it is a second time, and it is so grave, 
then I think it can lead to your dismissal (Prof. Yuvin).

The next item examined in this section is intercultural group work which is how students from different cultural backgrounds work together in small groups. There are often benefits and challenges that arise from such intercultural group work between students of different cultures (Lee et al., 2018; Liang, 2016). All BAME academics observed that students naturally gravitate towards other students from the same national, religious, or cultural origins, especially when asked to form small working groups. Such attitude is not beneficial to students as they will not be able to choose who to work or do business with after graduating from university. Hence, academics in this study often try to mix students up with others from distinct cultural or national backgrounds when working in small groups. However, even when students are mixed up, problems still arise, with some students failing to contribute, not attending meetings, or not doing their own part of the work. To solve these problems, an academic in this study reported that he often breaks the group into two between those who are not working and those who are working. Another academic said he puts a strategy in place where a person from one group explains to the other group what they have done and vice versa. Yet, a third academic said she would mark them individually, instead of marking them as a group, if she realizes that one person has worked harder that everyone else in the group.

\subsection{Context of Language}

The study of language has always had to deal with the difficult issue of representation (Kramsch, 1998). Data suggest that academics in this study, construct their social identity by categorizing themselves as belonging to a social group through representation (Thomas et al., 2004), but they also categorized students as belonging to different religious, racial or national groups. A typical example of representation is shown on the quote below by Mr. Anton.

I know in certain cultures, certain things could upset you, you know? Because I am very English, I have St. Georges flag on the wall, it was St. George's day yesterday. But I know that certain things might upset me, and a lot of things wouldn't upset me. We are not that easily upset. But something I did might upset you or might upset someone from say Somalia, or someone from Pakistan, and you know, just an action or not doing something quite right might upset completely unintentionally (Mr. Anton).

In this statement, Mr. Anton's choice of linguistic code, plays an important role in establishing his group identity (Thomas et al., 2004) as a typical, seemingly patriotic Englishman, who is proud of is heritage. He portrays the English as a people who do not easily get upset but also seem to suggest, from a completely 'outsider' perspective, that the interviewer, or people from other countries could easily get upset over minor things. This, in effect, is a classic view of categorization in representation. He assumes that all category members share certain important defining features. However, "for most social categories, categorization does not work in this way, although ironically, groups members may sometimes try to represent themselves like this" (Spencer-Oatey \& Franklin, 2009). Other examples of representation emerged from the data.

Once I thought accounting students and a Chinese student had the best mark (80\%), 
which is rare. And students were surprised that she cannot speak English how comes? I said, as I told them before, I don't mark the language. She used the right cases. This student was very succinct (Dr Rosaline).

Other students were surprised that a Chinese student scored $80 \%$ in the assignment because of their preconceived stereotypes about this Chinese student's language proficiency. They have wrongly associated the student's poor English proficiency to cognitive deficiency. Although the lecturer tried to defend this student, she too seemed to be surprised by saying that it was rare for a Chinese student to score that high. This is an unconscious bias that stems from representation. The next example of representation came from Dr Achal.

I thought a class of Chinese students. I went through the stages of a dissertation with them and none of them was asking any questions, and then I kept on saying do you understand? Should we move on? They just nodded. And then I asked myself if they were getting it. This is true. There are many aspects going into this. Is it shyness or they don't want to speak and get it wrong? Are they picking up the language? (Dr Achal)

We can see that Dr Achal asked several representational assumptive questions about Chinese students simply because they do not answer or ask questions. Again, these assumptions were made by him from an etic (outsider) perspective, but a Chinese lecturer teaching the same class would look at it from an emic (insider) standpoint. Many studies have suggested that the Confucian philosophy, with its emphasis on respect for authorities, can best explain Chinese students' behavior. Academics who are not familiar with this ideology would often make such assumptions when they teach Chinese students.

Another factor that emerged from the data regarding the context of language was the problem of accent. The term accent refers to pronunciation, and to speak with a regional accent for instance, is to pronounce words in a manner associated with a certain geographical area (Thomas et al., 2004). Both BAME and the British academic interviewed, identified accent as a major barrier to communication with students. The British lecturer admitted that he has had situations where students did not understand, not because of the language, but because of his accent. As a result, when teaching, he would make a conscious effort to slow his speech down.

...because when I am normally talking, I talk quite quickly because I am from Manchester. If I do that in front of the classroom, even those people who were born in this country don't understand me. So, people who are using English as a second language, haven't got hope of keeping up with the way we talk because we have got an accent which doesn't help. I notice it the opposite way around, when I have got students from certain areas, and they have got quite a strong accent (Mr. Anton).

He also struggles to understand certain students who speak with a 'strong' accent and that makes him think more about the students, for if he is struggling to understand them, there is a good chance they are struggling to understand him. Dr Stephen equally talked about the problem of accent.

Sometimes I teach a class of 80 students. And I have stood in front of the class and 
asked a question. There is a guy who is trying to answer the question, but I cannot even understand what he is saying. Not to embarrass the student, I must think of another direction of what he is trying to say, and it may not be exactly what they were trying to say (Dr Stephen).

He went on to explain how some students purposely do not talk in class because of their poor accents and that is frustrating as he wants his students to be talking to him. Just like Mr. Anton, he admitted that he believes students do not understand everything he says because of his nonnative accent. Although students have not complained about his accent, they have done so with other non-native lecturers. And sometimes, English lecturers have complained about the accents of foreign students. Dr. Stephen however, insisted that it is the duty of teachers to support the students and so they should not complain about students' accent but make effort to understand them. Dr. Rosaline on her part believes that students who are racist do pick on accent to discredit BAME lecturers, when they cannot pick on nothing else. She went further to say:

I am aware that I speak and pronounce differently, so, I always go slower. But students have said they understand me very well. Now I no longer apologize or say anything about my accent. I just go and teach, and I say if you don't understand any words, let me know.

I remember using BOREHOLE in class for a case, because that is what I read in textbooks and that is how I thought British students know. So, they kept asking what is borehole? Then I said in...we call it WELL. They said, but we call it WELL as well here. Sticking to the script sometimes is not good. They will understand you. Everybody has an accent. Scottish people have an accent, Liverpool people have an accent, everybody has an accent. So, we must try and understand each other (Dr Rosaline).

There was a consensus among academics that the best way to address the problem of differential accents, is to purposely speak slowly and to adjust to the student's way of speaking. This behavior is consistent with Giles' (2016), Communication Accommodation Theory- CAT. One of CAT's communicative strategies is convergence, that is, adjusting to ones' communicative behavior to be like another's' (Dragojevic 2016)

\subsection{Context of Academic Attainment}

All academics interviewed reported not noticing any gap in academic achievement between mainstream and BAME students. It was noted that local students have advantages over some BAME students in terms of grammar and academic writing, but they did not achieve better overall, academically. In fact, in many instances, it was reported that BAME students did much better than local students.

No, everyone performs pretty much the same. At the end of the day, they could all do the OSCE, they all pass exams. Our exams pass rate for the three that we did last year were straight across the board. It didn't matter where people are from (Mr. Anton). 
In terms of contents, good Indian students can be better than some home students. Some of them can perform very well and some of the home students can be failing while others are getting distinctions. But generally, in terms of expressions, grammar, and spellings, they have an edge (Dr Achal).

I think, sometimes, there could be more in terms of language, but to make a generalization that home students are better than foreign students- no. I wouldn't make that generalization. But sometimes there could be a difference because English is your first language, it is easier for you to understand and write well in English than someone who has a second language. In certain situations, you will find that this will happen, but thinking of it in terms of intellectual capacity, no. We don't have that kind of general situation that one can generalize. I think the language may be the only barrier (Prof. Yuvin).

My findings, therefore, do not support the statistics in the introduction (Universities UK, 2019) on the attainment gap between mainstream and BAME students in UK universities. Instead, my findings suggest that, apart from language advantage, there is no attainment gap between mainstream and BAME students in UK universities. In fact, one of my respondents unequivocally blamed the purported attainment gap on racism. She explained that some British lecturers intentionally mark down black and foreign students and that Chinese students are more scrutinized for cheating than British students.

We had a black student, who was very intelligent. He used to write about twenty cases. But my colleague will hardly ever give him a 70. My colleague once gave him 57 and I raised it to 70. I told him that he cannot give that paper 57. It was too good. They knew that I was always fighting for black students when I was there, and they didn't like that.

There is also this black Canadian student who had a problem with one of my colleagues because he accused her of not giving him enough feedback on his work. The lecturer reported him to the administration that he is threatening her life. Without any formal process nor investigation, they said they were going to expel him from the university, a third-year student. He told them he will sue them if they dared (Dr Rosaline).

\section{Discussion}

Despite a high intercultural awareness among academics, this study found that some academics and students still lacked the ability to negotiate reality (Antal \& Friedman, 2008) in an appropriate manner. This was evident by the fact that some students and academics were unable to deconstruct stereotypes in cultural representation. Their habitus seem to account for their behavior towards people from other cultures. For example, when Chinese students fail to speak in class or when Indian students stand up to greet the lecturer as he walks into the classroom. The experiences of academics in intercultural interaction with students were homogeneous across the board irrespective of their racial background. Accent was notably a significant 
barrier to intercultural communication and academics generally slowed down their speech to be made understood. This communication accommodation strategy was often cognitively motivated (Dragojevic 2016), in a bit to manage comprehension and communicative efficiency with students.

Despite their best efforts, universities do little to help academics and students develop and sustain intercultural awareness (Knohls \& Knight, 1994). In most cases, academics are left to figure out by themselves when confronted with cultural issues in the classroom. There is no systemic, structural, and coordinated approach in addressing cultural issues that may emerge in the classroom. Some BAME and mainstream students still lack the confidence and structure to engage in purposeful and substantive intercultural interaction (Lee et al., 2018). This study also found out that there was no attainment gap between mainstream and BAME students except for native-speaking language advantage that the former enjoys.

The major implication for policy in this study is that universities can draw lessons from here to improve and enhance their policies on diversity and multiculturalism. The study might also be useful to BAME academics who are planning to move to the UK for work, to understand the challenges and expectations of the job. Potential BAME academics living in the UK, who are planning to get into academia may find this study an important piece for their psychological preparation. This study recommends more intercultural training (Knohls \& Knight, 1994) for academics based on respect for knowledge and for learner's culture and communicative economy (Riley, 2007). This training should take the form of observation, sensitization, and negotiation of identities without forcing anyone to sacrifice their own ethos and cultural identity by being modelled in the image of another (Riley, 2007).

The limitation of this study is the small size of the sample. To make any generalization on these findings, a bigger sample size is required, and this is recommended for future studies. Future research might also want to study the relationship between racism and 'attainment gap', which is a factor that emerged strongly in my data.

\section{References}

Alexander, C., \& Arday, J. (2015). Aiming Higher: Race, Inequality and Diversity in the Academy. London, United Kingdom: Runnymede Trust.

Antal, A. B., \& Friedman, V. J. (2008). Learning to negotiate reality: A strategy for teaching intercultural competence. Journal of Management Education, 32(3), 363-386. https:// doi.org/10.1177/1052562907308794

Austin, J., \& Mathews, K. (2013). British Language \& Culture. London, United Kingdom: Lonely Planet Publications Pty Ltd.

Bell, J. (2005). Doing your Research Project: A Guide for first-time researchers in education, health, and social science. ( $4^{\text {th }}$ edition). Berkshire, United Kingdom: Open University Press. 
Berg, B. (2007). Qualitative Research Methods for the Social Sciences. Boston, USA: Pearson Education, Inc.

Bhopal, K. (2016). The experiences of Black and minority ethnic academics: a comparative study of the unequal academy. London and New York: Routledge.

Bryman, A. (2004). Social Research Methods. (2 ${ }^{\text {nd }}$ ed.). Oxford, United Kingdom: Oxford University Press.

Cotton, D. R. E., Joyner, M., George, R., \& Cotton, P. A. (2016). Understanding the gender and ethnicity attainment gap in UK higher education, Innovations in Education and Teaching International, 53(5), 475-486. https://doi.org/10.1080/14703297.2015.1013145

Denscombe, M. (2017). The good research guide for small-scale social research projects $\left(6^{\text {th }}\right.$ ed.). London, England: Open University Press.

Dragojevic, M., Gasiorek, J., \& Giles, H. (2016). Accommodative Strategies as Core of the Theory. In H. Giles (ed.). Communication Accommodation Theory: Negotiating Personal Relationships and Social Identities across Contexts (pp. 36-59). Cambridge, United Kingdom: Cambridge University Press.

Giles, H. (2016). Communication Accommodation Theory: Negotiating Personal Relationships and Social Identities across Contexts. Cambridge, United Kingdom: Cambridge University Press.

Gordon, C. (1980). Power Knowledge: Selected Interviews \& Other Writings by Michael Foucault. Brighton. United Kingdom: The Harvester Press.

Grenfell, M., James, D., Hodkinson, P., Reay, D., \& Robbins, D. (1998). Bourdieu and Education: Acts of Practical Theory. London, United Kingdom: Falmer Press Publishers.

Halbert, K., \& Chigeza, P. (2015). Navigating Discourse of Cultural Literacy in Teacher Education. Australian Journal of Teacher Education, 40, 155-168. https://doi.org/10.14221/ajte.2015v40n11.9

Hinkel, E. (1999). Culture in Second Language Teaching and Learning. Cambridge: Cambridge University Press.

Hui, S. K., \& Cheung, H. Y. (2014). Cultural literacy and student engagement: The case of technical and vocational education and training (TVET) in Hong Kong. Journal of Further and Higher Education, 39(4), 553-578. https://doi.org/10.1080/0309877X.2014.938263

Jones, J. (2004). Language and class. In L. Thomas, S. Wareing, I. Singh, J. S. Peccei, J. Thornborrow, \& J. Jones (Eds.), Language, Society and Power (pp. 133-155). London: United Kingdom: Routledge Taylor \& Francis Group.

Kidd, J. (2015). Representation. Oxfordshire, United Kingdom: Taylor \& Francis group.

Knohls, L. R., \& Knight, J. M. (1994). Developing Intercultural Awareness: A Cross-Cultural Training Handbook. (2nd ed.). Yarmouth, Maine, USA: Intercultural Press, Inc. 
Kramsch, C. (1998). Language and Culture. Oxford, United Kingdom; Oxford university press.

Lee, A., Poch, R., Smith, A., Delehanty K. M., \& Leopold, H. (2018). Intercultural pedagogy: A faculty learning cohort. Education Science, 8(4), 1-14. https://doi.org/10.3390/educsci8040177

Liang, M. (2016). Achieving multimodal cohesion during intercultural conversations. International Journal of Society, Culture \& Language, 4(2), 55-70.

Liddicoat, A. J., \& Crozet, C. (2000). Teaching Languages, Teaching Cultures. Melbourne Vic: Applied Linguistics Association of Australia.

Marsh, S. (2018, April 29). Cheating at UK top universities soar by 40\%. The Guardian. Retrieved from https://www.theguardian.com/education/2018/apr/29/cheating-at-top-ukuniversities-soars-by-30-per-cent/

McCulloch, G. (2018). BERA: Ethical guidelines for educational research (4th ed.). Retrieved from https://www.bera.ac.uk/researchers-resources/publications/ethical-guidelines-for educationalresearch-2018/

Muller, W. (2006). The contribution of 'cultural literacy' to the 'globally engaged curriculum' and the 'globally engaged citizen'. Social Educator, 24(2), 13-15.

Nchindia, C. A. (2020). An exploration of the effects of 'ESOL for citizenship' course on the sociocultural integration of adult learners into British society. International Journal of Society, Culture and Language, 8(2), 35-54.

Richardson, J. T. E. (2015). The under-attainment of ethnic minority students in UK higher education: what we know and what we don't know. Journal of Further and Higher Education, 39(2), 278-291. https://doi.org/10.1080/0309877X.2013.858680

Riley, P. (2007). Language, Culture, and Identity. London, United Kingdom: Continuum.

Sian, K. P. (2017). Being Black in a White World: Understanding Racism in British Universities. Papeles del Centro de Estudios sobre la Identidad Colectiva, 176, ISSN 1695-6494.

Skutnabb-Kangas, T., \& Cummins, J. (1988). Minority education: from shame to struggle. Clevedon, England: Multilingual Matters Ltd.

Spencer-Oatley, H., \& Franklin, P. (2009). Intercultural interaction: A multidisciplinary approach to intercultural communication. Basingstoke, Hampshire: Palgrave Macmillan.

Scallon, S. (1999). Not to waste words or students: Confucian and Socratic discourse in the tertiary classroom. In E. Hinkel (Ed.)., Culture in second language teaching and learning (pp. 13-26). Cambridge, United Kingdom: Cambridge University Press.

Thomas, B., Jack, N., Allerhand, M., \& Deary, I. (2014). Does bilingualism influence cognitive aging? Annals of neurology, 75(6), 959-963. https://doi.org/10.1002/ana.24158

Thomas, L., Wareing, S., Singh, I., Peccei, J. S., Thornborrow, J., \& Jones, J. (1999). Language, Society and Power (2nd ed.). London, United Kingdom: Routledge. 


\section{Macrothink}

Universities, UK. (2019). Black, Asian, and Minority Ethnic student attainment at UK universities: closing the gap. Retrieved from https:/www.universitiesuk.ac.uk/closingthe-gap/

\section{Acknowledgment}

I am grateful to the five academics who participated in this study. Although their names have been pseudonymized throughout this paper for confidentiality, this study would not have been possible without them.

\section{Copyright Disclaimer}

Copyright for this article is retained by the author(s), with first publication rights granted to the journal.

This is an open-access article distributed under the terms and conditions of the Creative Commons Attribution license (http://creativecommons.org/licenses/by/3.0/). 